\title{
Avaliação Multidimensional Baseada em Lógica Difusa para Ambientes de Ensino Mediado por Computador
}

\author{
Multidimensional Evaluation based on Fuzzy Logic for e-Learning Environments
}

Richard A. Arias

Universidade Estadual de Campinas (UNICAMP)

Faculdade de Engenharia Elétrica e Computação

darrich@gmail.com

Gean D. Breda

Universidade Estadual de Campinas (UNICAMP)

Faculdade de Engenharia Elétrica e Computação

gdbreda@gmail.com

Leonardo de S. Mendes

Universidade Estadual de Campinas (UNICAMP)

Faculdade de Engenharia Elétrica e Computação

$\underline{\text { lmendes@decom.fee.unicamp.br }}$

\author{
André M. Panhan \\ Universidade Estadual de Campinas (UNICAMP) \\ Faculdade de Engenharia Elétrica e Computação \\ apanhan@gmail.com \\ Bruno B. Zarpelão \\ Universidade Estadual de Londrina (UEL) \\ Departamento de Computação \\ brunozarpelao@gmail.com
}

\begin{abstract}
Resumo Diferentes formas de avaliar o desempenho de estudantes vêm sendo pesquisadas nas últimas décadas. Este artigo assume a multidimensionalidade da avaliação de desempenho como um resultado de interações complexas. Assim, adota uma metodologia para avaliação baseada em princípios da lógica difusa. As etapas da metodologia estabelecida constituem um guia para o desenvolvimento de uma avaliação multidimensional capaz de aferir o aprendizado dos estudantes em ambientes mediados por computador. Para o desenvolvimento da pesquisa, foi necessário contextualizar as teorias, os modelos e sistemas utilizados na avaliação do desempenho dos alunos.
\end{abstract}

Palavras-Chave: Avaliação Educacional; Sistemas Difusos; Lógica Difusa; Desempenho Escolar; Ensino Mediado por Computador.

\begin{abstract}
The ways of evaluating the performance of students have been exhaustively sought in the last decades. This paper understands the performance evaluation multidimensionality as a result of complex interactions. Therefore, it adopts an evaluation methodology based on fuzzy logic principles. The methodology steps make a guide for the development of a multidimensional evaluation capable of gauging the student learning in e-learning environments. For the development of the research, it was necessary to put the conditions into context of the theories, the models and systems utilized in the evaluation of students.
\end{abstract}

Keywords: Educational Evaluation; Fuzzy Systems; Fuzzy Logic; Scholar evaluation; Computer Mediated Learning Environments 



\section{Introdução}

Nos últimos dez anos, pesquisadores como Weon e Kim[9], Bai e Chen [1][2] e Saleh e Kim [7] tem utilizado a teoria dos conjuntos difusos na resolução de problemas em modelos de avaliação de desempenho de estudantes. Os conjuntos difusos foram introduzidos por $\mathrm{Za}$ deh[10], baseados na ideia de que existem situações nas quais não está claro se um elemento pertence ou não a um determinado conjunto. Nos conjuntos clássicos, a relação de pertinência entre o elemento e o conjunto é definida como falsa (0) ou verdadeira (1). Nos conjuntos difusos, a relação de pertinência entre o elemento e o conjunto está definida entre os valores de 0 e 1 .

Neste artigo, propomos adotar uma metodologia para avaliação multidimensional de desempenho baseada em princípios da lógica difusa, contemplando os três critérios definidos por Weon e Kim [9], a dificuldade, a importância e a complexidade das questões para ambientes de ensino mediados por computador [6][8]. Para a implementação da metodologia de avaliação multidimensional em ambientes de ensino mediados por computador foi desenvolvido um modelo de avaliação difusa utilizando três Fuzzy Logic Controller (FLC) de forma a representar os três critérios de avaliação mencionados por Weon e Kim [9], Bai e Chen [2] e Saleh e Kim [7]. O trabalho está assim organizado. No Seção 2 introduzimos os conceitos teóricos necessários ao entendimento da metodologia. No Seção 3 introduzimos a avaliação multidimensional em si. A seção 4 mostra o estudo de caso que foi desenvolvido. Por último temos a Conclusão e as Referências Bibliográfica.

\section{Fundamentos Teóricos}

A avaliação de aprendizado deve gerar subsídios aos professores, oferecendo condições de determinar quais pontos devem ser reforçados no processo de ensinoaprendizagem do aluno. É igualmente importante uma avaliação condizente com o esforço empregado pelos alunos, evitando prejudicá-los em futuras seleções que sejam baseadas no aproveitamento acadêmico [7].

\subsection{Método de Weon e Kim}

Weon e Kim [9] desenvolveram um método de avaliação de desempenho baseado em graus de pertinência aos conjuntos, pesos dos fatores determinados por um especialista no domínio e funções matemáticas para avaliação. Neste método são considerados três fatores relevantes para realizar uma avaliação: a dificuldade, a importância e a complexidade das questões. Os autores avaliaram os acertos das respostas considerando individualmente os três fatores mencionados e os limites de tempo estabelecidos para cada questão.
$\mathrm{Na}$ primeira etapa, os autores determinam o resultado parcial de desempenho dos alunos mediante a avaliação individual de cada questão, empregando para isso funções de pertinência difusa definidas pelos próprios autores. Nesta etapa é calculada a pontuação final de cada questão, por meio da soma dos pontos das subquestões. A pontuação final das questões é normalizada, aplicando-se média aritmética na pontuação das subquestões associadas. Para finalizar esta primeira etapa, os valores normalizados obtidos serão avaliados nas funções de pertinências difusas, associadas aos conjuntos difusos definidos pelo domínio das questões.

$\mathrm{Na}$ segunda etapa, os autores determinam o resultado final da avaliação de desempenho dos alunos por meio da soma de pontos de cada questão. O resultado obtido será normalizado, aplicando a média aritmética das subquestões associadas a cada questão. Os valores normalizados obtidos serão avaliados nas funções de pertinências associadas a cada conjunto difuso. O resultado desta avaliação determina o desempenho do aluno, baseado no critério de avaliação utilizado: o limite de tempo, a importância, a complexidade e a dificuldade das questões.

Porém, o método de Weon e Kim [9] apresenta como problema a subjetividade na atribuição de pesos para o fator de dificuldade, no qual os valores são determinados por um especialista no domínio.

\subsection{Método de Bai e Chen}

Bai e Chen [2], Figura 1, propuseram um método que utiliza as funções de pertinência e regras difusas para determinar o desempenho de alunos. O grau de dificuldade foi calculado em função do número de acertos e o tempo gasto para responder às questões. Desta forma, os autores tentaram solucionar a subjetividade do fator de dificuldade proposto no método de Weon e Kim [9]. Já, os fatores de complexidade e a importância no método de Bai e Chen [2] são determinados por um especialista no domínio.

O método proposto pelos autores inicia com o cálculo da pontuação dos alunos em cada questão formando a Matriz de Acertos. Em seguida, é elaborada a Matriz de Tempos, formada a partir do tempo gasto pelos alunos na resolução das questões. Uma terceira matriz contém a pontuação máxima das questões, chamada Matriz de Pesos. Os valores definidos pelos especialistas no domínio para a importância e a complexidade das questões, serão armazenados na Matriz de Importância e na Matriz de Complexidade, respectivamente. Por fim, os autores definem as funções de pertinência difusas associadas aos cinco conjuntos difusos: "Baixo", "Baixo-Médio", "Médio", "Médio-Alto" e "Alto".

Inicialmente, Bai e Chen [2] calculam as notas com base apenas no cruzamento da Matriz de Acertos com a 
Matriz de Pesos. Contudo, esta solução poderá gerar alunos com a mesma pontuação. Assim, o método proposto pelos autores reclassifica os alunos com a mesma pontuação, mediante a obtenção de valores de ajuste.
Para isso, os autores utilizam funções de pertinências e regras difusas, considerando a dificuldade, a complexidade e a importância das questões.

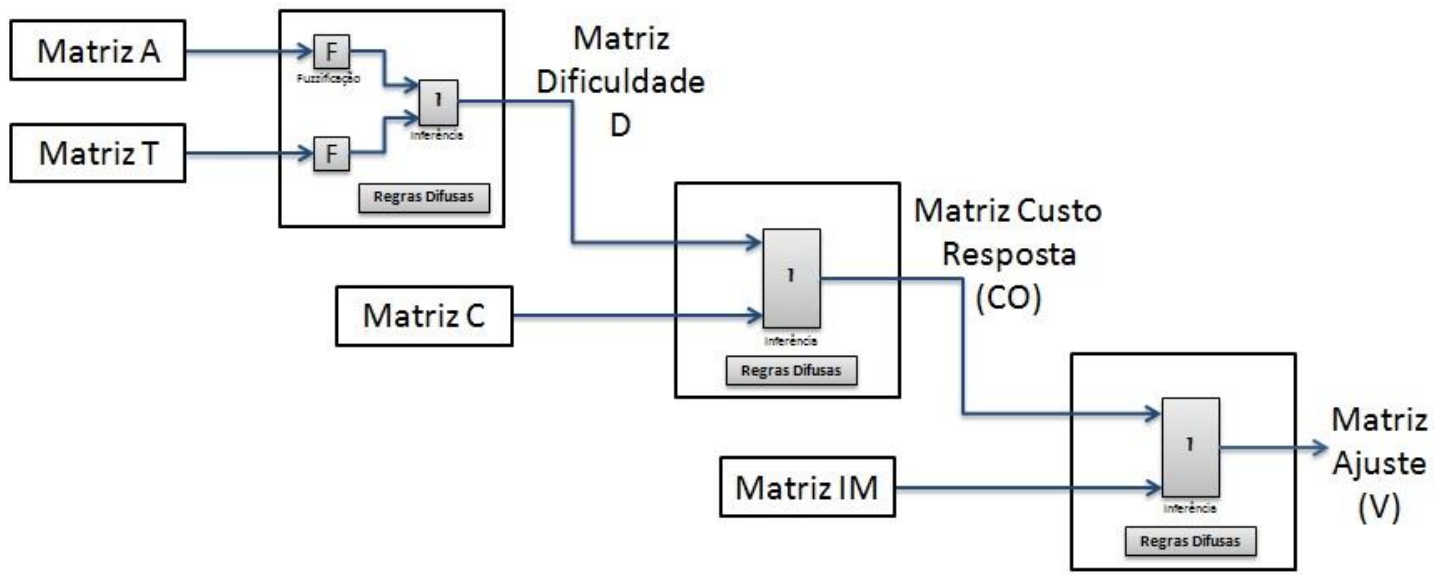

Figura 1 : Modelo de Bai e Chen (2008)

Os autores executam a inferência difusa sobre a Matriz de Acertos e a Matriz de Tempos utilizando as regras difusas associadas a estas matrizes. Como resultado obtém-se a Matriz de Dificuldade das questões. Com a Matriz de Dificuldade e a Matriz de Complexidade, executa-se a inferência difusa utilizando as regras difusas correspondentes a estas matrizes. O resultado é a Matriz de Esforço. Por fim, executa-se a inferência difusa sobre a Matriz de Esforço e a Matriz de Importância, obtendo o ajuste dos pesos das questões.

Com os valores de ajuste, os autores calcularam os valores finais de ajuste necessários para cada questão, utilizando para isso os valores de defuzzificação associados aos conjuntos difusos. Baseado nos valores finais de ajuste, os autores calcularam uma nova pontuação para os alunos que obtiveram a mesma pontuação na classificação tradicional, utilizando a fórmula chamada Soma da Diferença. A nova pontuação calculada permitiu reclassificar os alunos, eliminado o empate.

No entanto, o método de Bai e Chen [2] ainda apresenta como problema a subjetividade na atribuição de pesos para os fatores de ponderação, pois os fatores de complexidade e importância também foram determinados por um especialista no domínio.

\subsection{Método de Saleh e Kim}

Saleh e Kim [7] propuseram uma alternativa ao método de Bai e Chen [2]. A proposta dos autores é um sistema de avaliação do desempenho de alunos baseada na lógica difusa considerando três fatores: a dificuldade, a complexidade e a importância das questões, como o método proposto por Weon e Kim [9]. Porém, o trabalho de
Saleh e Kim [7] se baseia no método de inferência difusa de Mamdani [5] e no método de defuzzificação denominado Center of Gravity (COG) [4].

O sistema proposto por Saleh e Kim [7] é representado como um diagrama de blocos de nós difusos como apresentamos na Figura 2.

O sistema consiste em três nós: dificuldade, custos e ajuste. Cada nó do sistema se comporta como um controlador difuso - Fuzzy Logic Controller (FLC) - com duas entradas e uma saída, como podemos observar na Figura 3. Neste sistema os mapas FLC apresentam uma relação difusa de dois para um, por inferência através de uma regra base.

Para determinar a dificuldade, o esforço e o ajuste das questões, estes controladores estão interligados sequencialmente, onde a saída do primeiro controlador FLC, chamado nó de dificuldade, é então a entrada do segundo controlador FLC chamado nó de esforço. A saída do controlador nó de esforço é então a entrada do ultimo controlador FLC chamado nó de ajuste.

A saída do método de Saleh e Kim [7] é o nó de ajuste, denominado Vetor de Ajuste (W) com os novos pesos das questões, utilizados para obter a nova classificação dos alunos.

O diagrama de blocos apresentado pelo Saleh e Kim [7] é composto por:

- Dados de entrada - Dos alunos, a Matriz Taxa de Acertos A, Matriz Taxa de Tempo T. Do especialista no domínio das questões, a Matriz de 
Complexidade $C$, a Matriz de Importância $P$ e o Vetor de Pesos Máximos das questões $G$.

- Processo de avaliação - Composto pelo nó de dificuldade $\left(F L C_{D}\right)$, o nó de esforço $\left(F L C_{E}\right)$ e o nó de ajuste $\left(F L C_{W}\right)$, onde cada nó comporta-se como um controlador lógico difuso.

- Dados de saída - É o vetor que contém os valores de ajuste final requerido $(W)$.

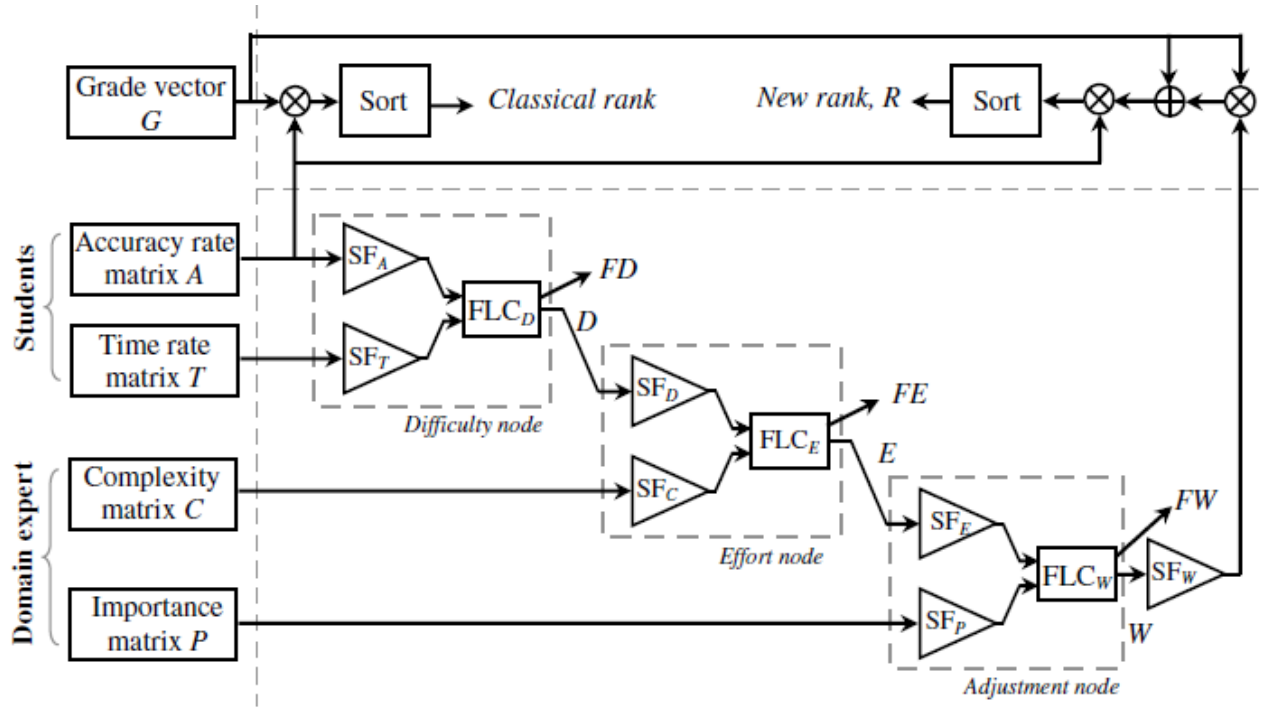

Crisp world

Fuzzy world

Figura 2: Diagrama do sistema de avaliação difuso de Saleh e Kim (2009).

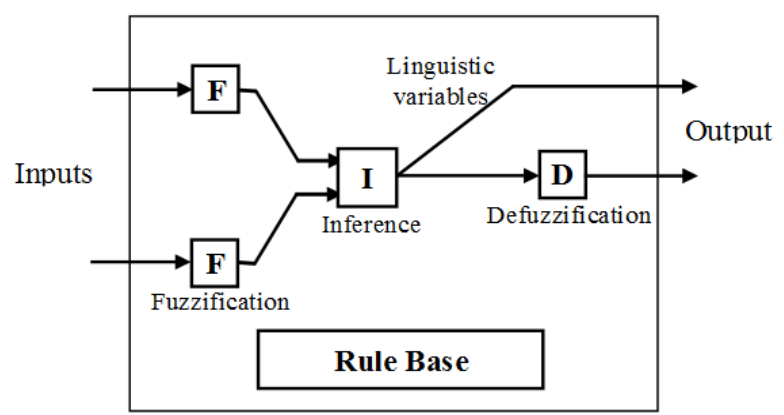

Figura 3: Representação do nó como um controlador difuso

No diagrama de blocos, observam-se os fatores de escalas $\mathrm{SF}_{\mathrm{s}}$ nas entradas de cada nó, cujos valores podem ser alterados a critério do avaliador, influenciando os valores de saída de cada nó.

\subsection{Analise dos Métodos}

Na Tabela 1 no método de Weon e Kim [9] vemos que ele não utiliza os processos de fuzzificação e defuzzificação, apresentando como problema principal a subjetividade na atribuição de pesos para os fatores de complexidade e importância.

Já o método proposto por Bai e Chen [2] utiliza como processo de inferência o modelo de Máximo e Pesos e como processo de defuzzificação o modelo de Valores. O processo de fuzzificação foi baseado no método triangular. Já o grau de dificuldade foi calculado em função do número de acertos e o tempo gasto para responder às questões. Desta forma, os autores tentaram solucionar a subjetividade do fator de dificuldade proposto no método de Weon e Kim [9]. Porém, os fatores de complexidade e a importância no método de Bai e Chen [2] são determinados por um especialista no domínio.

\begin{tabular}{|c|c|c|c|}
\cline { 2 - 4 } \multicolumn{1}{c|}{} & $\begin{array}{c}\text { Fuzzifica- } \\
\text { ção }\end{array}$ & Inferência & $\begin{array}{c}\text { Defuzzifica- } \\
\text { ção }\end{array}$ \\
\hline $\begin{array}{c}\text { Weon e } \\
\text { Kim }\end{array}$ & - & $\begin{array}{c}\text { Funções de } \\
\text { Avaliação }\end{array}$ & - \\
\hline $\begin{array}{c}\text { Bai e } \\
\text { Chen }\end{array}$ & Método & Máximos e & $\begin{array}{c}\text { Valores de } \\
\text { Defuzzifica- } \\
\text { ção }\end{array}$ \\
\hline $\begin{array}{c}\text { Saleh } \\
\text { e Kim }\end{array}$ & Mriangular & Mamdani's & $\begin{array}{c}\text { Centro de } \\
\text { Travidade } \\
\text { (CoG) }\end{array}$ \\
\hline
\end{tabular}

Tabela 1: Quadro comparativo dos métodos 
Já o método de Saleh e Kim [7] utiliza como processo de inferência o modelo de Mamdani [5] e um método de defuzzificação denominado Center of Gravity (COG) [4]. O processo de fuzzificação foi baseado no método triangular. A transparência e o objetivo do sistema fuzzy o tornam um método de fácil compreensão para professores. A adoção de funções de pertinências triangulares convencionais pode ser alterada a critério do avaliador de forma a influenciar os valores de saída de cada nó.

\section{Avaliação Multidimensional}

Neste trabalho, aplicamos os conceitos de Weon e Kim [9], Bai e Chen [2] e, também, nos baseamos no modelo de Saleh e Kim [7] para a avaliação do desempenho de alunos em ambientes de ensino mediados por computador. O modelo adotado tem como entrada de dados o Vetor de Peso das Notas, a Matriz Taxa de Acertos, a Matriz Taxa de Tempos, a Matriz de Complexidade e a Matriz de Importância. O processo de avaliação difusa sobre os dados de entrada é realizado por três Fuzzy Logic Controller (FLC), que internamente executam a fuzzificação, a inferência e defuzzificação dos valores de entrada junto com as regras difusas determinadas por um especialista no domínio. Como dados de saída obtemos um Vetor de Pesos ajustados, permitindo avaliar o desempenho dos alunos de forma multidimensional.

\subsection{Modelo de avaliação multidimensional}

O modelo adotado está baseado nos três critérios de avaliação citados pelo Weon e Kim [9]: bloco de dificuldade, bloco de esforço e bloco de ajuste, ver Figura 4. Este foi concebido baseado no modelo de Saleh e Kim [7], onde adotamos o método triangular para realizar a fuzzificação. O processo de inferência adota o modelo de Mandani's [5] e o de defuzzificação adota o método do Center of Gravity (COG) de Lancaster e Wierman [4].

O bloco de dificuldade recebe como entrada a Matriz Taxa de Acertos e a Matriz Taxa de Tempos obtidos pelos alunos na resolução das atividades educacionais propostas. A saída deste bloco vai indicar a dificuldade do aluno na resolução das atividades educacionais de acordo com o tempo gasto.

O bloco de esforço recebe como entrada a Matriz de Dificuldade e a Matriz de Complexidade. Sendo que a Matriz de Complexidade é determinada por um especialista no domínio. A saída deste bloco vai indicar o esforço despendido pelo aluno na resolução das atividades educacionais, considerando a dificuldade e complexidade das mesmas.O bloco de ajuste recebe como entradas a Matriz de Esforço e a Matriz de Importância, sendo que
Matriz de Importância é determinada por um especialista no domínio. A saída do terceiro bloco será a Matriz de Ajuste, contendo os novos valores dos pesos das notas das atividades educacionais.

Os três Fuzzy Logic Controller (FLC) permitem definir os novos pesos das atividades educacionais, conforme apresentado na Figura 4.

Os novos valores dos pesos das notas das atividades educacionais representam o estado exato dos elementos associados ao processo da avaliação. Estes valores são as Matrizes de Acertos, Tempos, Complexidade e Importância das atividades.

No modelo adotado descrevemos cada uma das entradas como segue:

- Vetor de Peso da Nota: é um vetor que contém os valores de pontuação máximos para cada atividade. Estes valores devem ser definidos por um especialista no domínio. A soma de todos eles deve ser igual a cem.

- Matriz de Taxa de Acertos: é uma matriz que contém a taxa de acertos dos alunos nas atividades educacionais. Esta matriz deve ser obtida mediante consulta da média histórica de acertos dos alunos.

- Matriz de Tempos: é uma matriz que contém os tempos obtidos pelos alunos na resolução das atividades educacionais.

- Matriz de Complexidade: é uma matriz que contém os pesos de complexidade das atividades educacionais, definidos por um especialista no domínio.

- Matriz de Importância: é uma matriz que contém os pesos de importância das atividades educacionais, também definidos por um especialista no domínio.

A partir do modelo apresentado, o processo de avaliação do desempenho dos alunos na resolução das atividades educacionais ocorre em três etapas.

$\mathrm{Na}$ primeira etapa o professor responsável pelas atividades educacionais proposta deverá cadastrar os pesos de complexidade e importância para as questões que compõem as atividades. Na segunda etapa, os alunos solucionam as questões das atividades educacionais propostas. A taxa de acertos e o tempo gasto pelos alunos para cada uma das questões deverão ser armazenados. Já na terceira etapa os dados obtidos serão inseridos no modelo difuso de avaliação multidimensional, representado na Figura 4. 


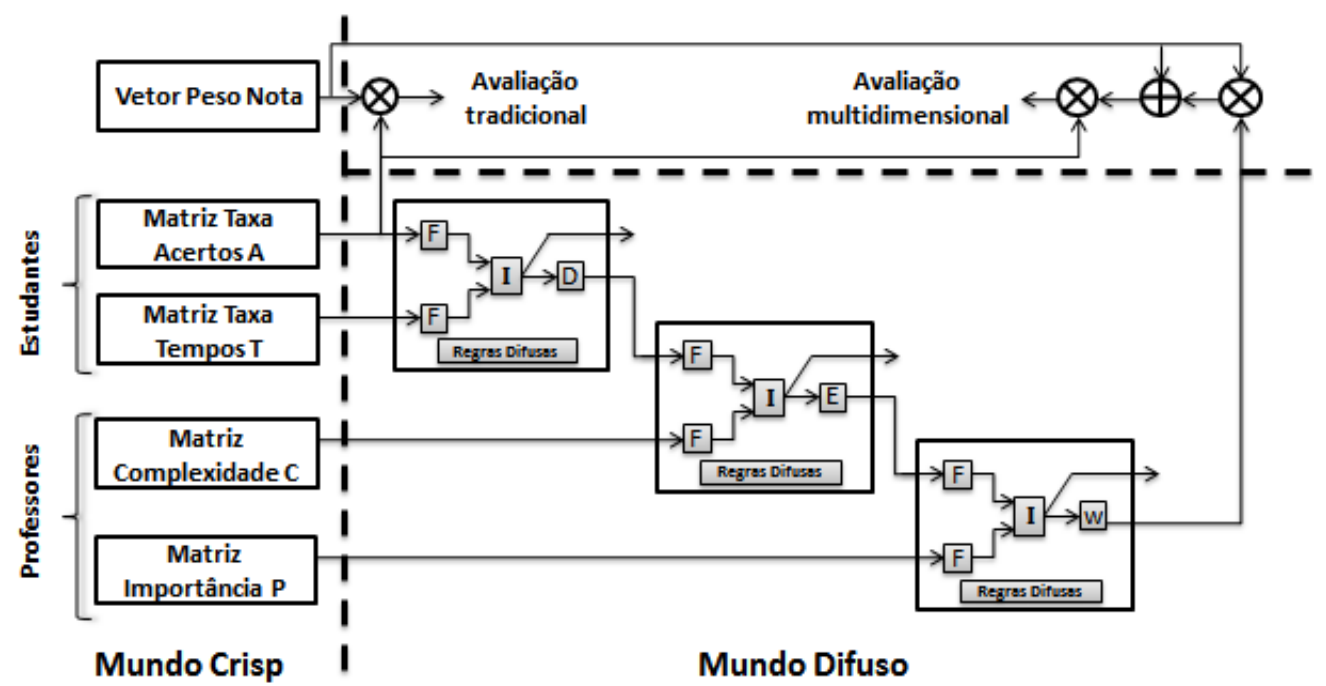

Figura 4: Diagrama do sistema de avaliação difuso multidimensional adptado de Saleh e Kim (2009)

Estes dados foram utilizados como entrada do bloco dificuldade. O especialista no domínio define as Matrizes de Complexidade e Importância para as atividades educacionais. Estas matrizes serão as entradas dos blocos de esforço e de ajuste respectivamente.

A saída do bloco de ajuste do modelo adotado é um vetor contendo os valores dos novos pesos das atividades.

\subsection{Implementação do Modelo}

Para a implementação do modelo foi utilizado o MatLab. A Figura 5 apresenta o bloco de dificuldade, constituído por um Fuzzy Logic Controller (FLC) com suas respectivas regras.

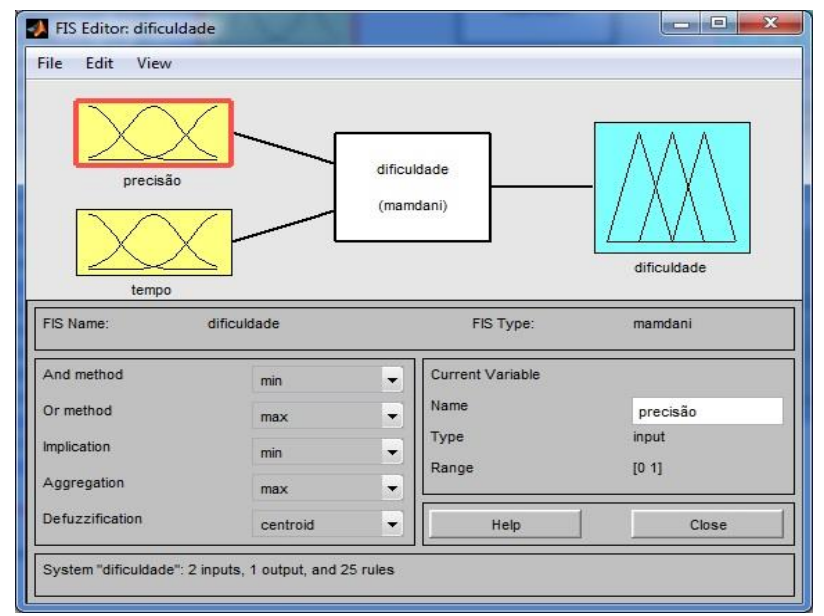

Figura 5: FIS Editor para construção de FLC no MatLab

O editor para FLC do MatLab permite a fuzzificação, a inferência e defuzzificação dos valores de entrada junto com as regras difusas que são definidas por um especia- lista no domínio nas atividades educacionais. Para criação dos conjuntos difusos que representam as variáveis de entrada foram utilizados os editores de funções de pertinência imerso dentro de cada FLC do MatLab Já o editor de regras difusas do MatLab permite inserir as regras difusas que representam o conhecimento do especialista e servem para inferir a saída do FLC.

\section{Estudo de Caso}

Para avaliar o modelo adotado, aplicamos a avaliação multidimensional baseada em lógica difusa nos resultados obtidos pelos alunos da escola Aletheia, uma escola da rede privada do município de Campinas,

A escola Aletheia adotou nos últimos anos o ensino mediado por computador como ferramenta para o processo ensino-aprendizagem, buscando garantir a eficácia e o sucesso dos cursos. Assim, escolheram como ferramentas pedagógicas para promover o ensino mediado por computador o software educacional Conexão do Saber [3].

O software educacional Conexão do Saber se originou de uma participação do Laboratório de Redes de Comunicação (LaRCom) da Faculdade de Engenharia Elétrica e de Computação (FEEC) da Universidade Estadual de Campinas (UNICAMP) no projeto Partnership in Global Learning (PGL). O projeto estabeleceu um programa de colaboração entre a Universidade da Flórida (UF/USA), a Universidade Estadual de Campinas (UNICAMP/Brasil), a Pontifícia Universidade Católica do Rio de Janeiro (PUC-RJ/Brasil), a Fundação Getúlio Vargas (FGV/Brasil) e o Instituto de Tecnologia de Monterrey (ITESM/México). 
Os principais objetivos do projeto Conexão do Saber são: o desenvolvimento de conteúdos didáticos multimídia, chamados módulos; o desenvolvimento de laboratórios virtuais; o estudo e implantação do sistema para gerenciamento e distribuição dos conteúdos via Internet, a formação de professores do Ensino Fundamental e de técnicos em informática.

O núcleo do Conexão do Saber é formado por módulos educacionais. Eles são definidos como conjunto de atividades interativas e lúdicas com o intuito de comple- mentar e/ou avaliar um conteúdo já desenvolvido com os alunos em sala de aula. Uma disciplina é composta por vários módulos e cada módulo é composto por várias atividades. A Figura 6 apresenta os elementos de um módulo educacional [3].

Os dados utilizados neste estudo de caso foram coletados durante a avaliação de 10 alunos do quinto ano na utilização dos módulos educacionais de matemática, compostos por 7 atividades educacionais.

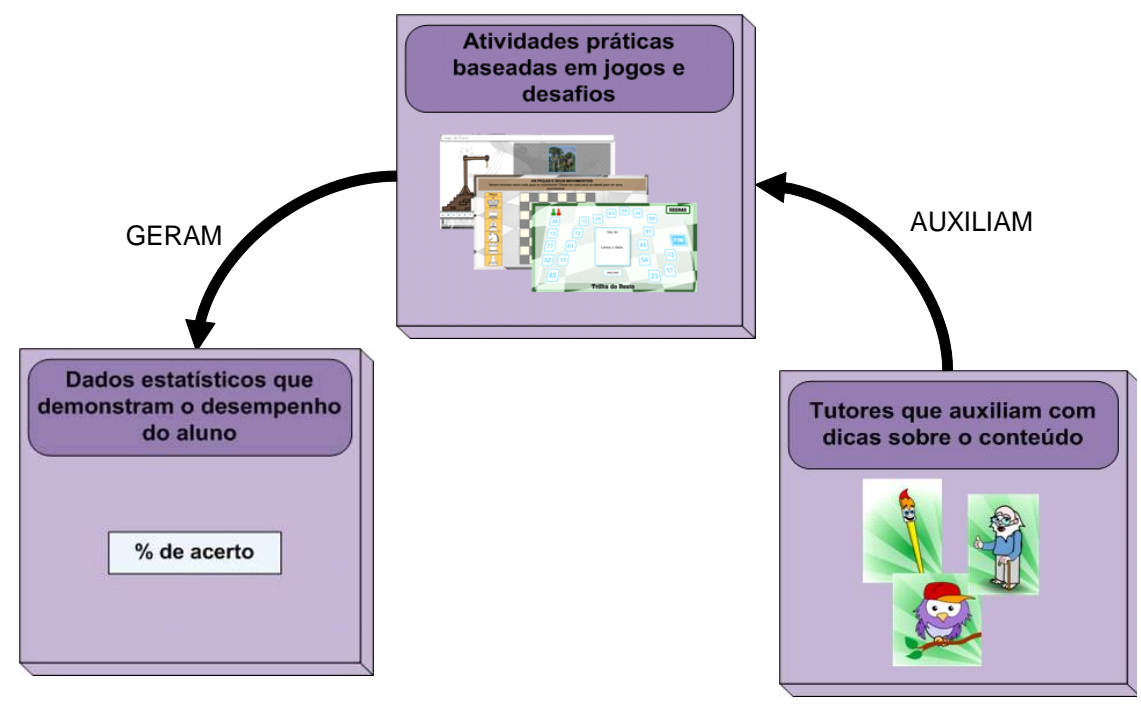

Figura 6: Elementos do módulo educacional (Inocêncio 2011).

\subsection{Obtenção de dados de entrada}

Os acertos dos alunos foram armazenados no sistema Conexão do Saber, já os tempos gastos para resolução das atividades foram coletados de forma manual, já que o sistema não armazena os tempos em que os alunos permaneceram nas atividades. Tanto os acertos quanto os tempos, foram organizados nas matrizes Taxa de Acertos e Tempos, respectivamente.

A Matriz Taxa de Acertos $A$ é composta por valores entre 0 e 1 . Assim, quando o aluno acertar uma atividade completamente $(100 \%)$, o valor associado à Matriz de Acertos será " 1,0 ", já se o aluno acertar a atividade parcialmente $(50 \%)$, o valor associado à matriz de acertos será “ 0,5 " e para o aluno que não realizar uma atividade $(0 \%)$, o valor associado à Matriz de Acertos será de “ 0,0 ”.

A Matriz de Tempos $T$ representa o tempo utilizado pelo aluno na resolução da atividade. O valor do tempo utilizado pelos alunos foi obtido em segundos e dividido pelo maior tempo obtido na atividade.

Os valores das Matrizes de Pesos $G$, da Matriz de Complexidade $C$ e da Matriz de Importância $P$ foram definidos por um especialista no domínio, um professor da disciplina de matemática.

A Matriz de Pesos $P$ foi composta pelos pesos máximos de cada atividade do módulo educacional, a soma desses pesos deve totalizar 100 .

A Matriz de Complexidade $C$ e a Matriz de Importância $P$ foram construídas mediante pesos relativos, definidos por um especialista no domínio. Os valores compreendidos entre 0 e 1 , para cada atividade do módulo educacional avaliado.

\subsection{Módulo educacional avaliado}

Para este estudo de caso foi avaliado o desempenho dos alunos da quinta série do ensino fundamental no módulo de Números Naturais. A Matriz de Acertos, Tempos, Complexidade, Importância e Pesos máximos obtidos para o módulo "Números Naturais" da disciplina de matemática da quinta série, são apresentadas nas tabelas $2,3,4,5$ e 6 . 


\begin{tabular}{|l|c|c|c|c|c|c|c|c|c|c|}
\multicolumn{10}{|c|}{} \\
\cline { 2 - 11 } & ALUNO1 & ALUNO2 & ALUNO3 & ALUNO4 & ALUNO5 & ALUNO6 & ALUNO7 & ALUNO8 & ALUNO9 & ALUNO10 \\
\hline ATIV1 & 1,0 & 1,0 & 0,5 & 0,5 & 0,5 & 0,5 & 1,0 & 1,0 & 1,0 & 1,0 \\
\hline ATIV2 & 1,0 & 1,0 & 1,0 & 1,0 & 0,0 & 1,0 & 1,0 & 1,0 & 1,0 & 1,0 \\
\hline ATIV3 & 1,0 & 1,0 & 1,0 & 1,0 & 1,0 & 1,0 & 1,0 & 1,0 & 1,0 & 1,0 \\
\hline ATIV4 & 0,0 & 0,0 & 0,0 & 0,0 & 0,0 & 0,0 & 0,0 & 0,0 & 0,5 & 0,0 \\
\hline ATIV5 & 0,0 & 1,0 & 0,0 & 0,0 & 0,0 & 0,0 & 0,0 & 0,0 & 0,0 & 0,0 \\
\hline ATIV6 & 1,0 & 1,0 & 1,0 & 0,0 & 0,0 & 0,0 & 0,0 & 0,0 & 1,0 & 1,0 \\
\hline ATIV7 & 0,0 & 0,5 & 0,5 & 0,5 & 0,5 & 0,0 & 0,0 & 0,0 & 0,5 & 0,0 \\
\hline
\end{tabular}

Tabela 2: Matriz de Acerto do módulo "números naturais" da quinta série".

\begin{tabular}{|l|l|l|l|l|l|l|l|l|l|l|}
\hline \multicolumn{1}{|c|}{} & \multicolumn{7}{|c|}{ TEMOS } \\
\hline & ALUN01 & ALUNO2 & ALUN03 & ALUNO4 & ALUNO5 & ALUNO6 & ALUNO7 & ALUNO8 & ALUNO9 & ALUNO10 \\
\hline ATIV1 & 0,3259 & 0,2567 & 0,3304 & 0,3616 & 0,1942 & 0,0536 & 0,4554 & 1,0000 & 0,2411 & 0,2835 \\
\hline ATIV2 & 0,2209 & 0,4186 & 0,3488 & 0,3140 & 0,1047 & 0,3837 & 0,3488 & 1,0000 & 0,2674 & 0,3023 \\
\hline ATIV3 & 0,6400 & 0,4500 & 1,0000 & 0,5000 & 0,5200 & 0,4500 & 0,7000 & 0,8700 & 0,5700 & 0,7700 \\
\hline ATIV4 & 0,3406 & 0,2089 & 0,6498 & 0,7029 & 0,0809 & 0,1498 & 1,0000 & 0,8176 & 0,2935 & 0,1763 \\
\hline ATIV5 & 0,7088 & 0,3123 & 0,0977 & 0,1590 & 0,0479 & 0,3851 & 0,4406 & 0,0594 & 0,3448 & 1,0000 \\
\hline ATIV6 & 0,2662 & 0,2922 & 0,5519 & 1,0000 & 0,2727 & 0,1753 & 0,0000 & 0,0000 & 0,3442 & 0,7273 \\
\hline ATIV7 & 0,4082 & 0,7461 & 0,3763 & 0,2995 & 1,0000 & 0,1909 & 0,0000 & 0,0000 & 0,6374 & 0,3068 \\
\hline
\end{tabular}

Tabela 3: Matriz de Tempo do módulo "números naturais" da quinta série".

\begin{tabular}{|c|c|c|c|c|c|c|}
\hline \multicolumn{7}{|c|}{ COMPLEXIDADE } \\
\hline ATIV1 & ATIV2 & ATIV3 & ATIV4 & ATIV5 & ATIV6 & ATIV7 \\
\hline 0,3 & 0,4 & 0,4 & 0,6 & 0,6 & 0,6 & 0,6 \\
\hline
\end{tabular}

Tabela 4: Matriz de Complexidade do módulo "Números Naturais" da quinta série".

\begin{tabular}{|c|c|c|c|c|c|c|}
\hline \multicolumn{7}{|c|}{ IMPORTANCIA } \\
\hline ATIV1 & ATIV2 & ATIV3 & ATIV4 & ATIV5 & ATIV6 & ATIV7 \\
\hline 0,4 & 0,4 & 0,5 & 0,6 & 0,6 & 0,6 & 0,6 \\
\hline
\end{tabular}

Tabela 5: Matriz de Importância do módulo "Números Naturais" da quinta série".

\begin{tabular}{|c|c|c|c|c|c|c|}
\hline \multicolumn{7}{|c|}{ PESOS DAS ATIVIDADES } \\
\hline ATIV1 & ATIV2 & ATIV3 & ATIV4 & ATIV5 & ATIV6 & ATIV7 \\
\hline 5 & 15 & 10 & 20 & 15 & 15 & 20 \\
\hline
\end{tabular}

Tabela 6: Matriz de Pesos do módulo "Números Naturais" da quinta série. 


\subsection{Obtenção de Regras Difusas}

As regras difusas fazem parte do motor de inferência difuso e também são determinados por um especialista no domínio, neste caso um professor da disciplina de matemática, para os três fatores de avaliação mencionados por Weon e Kim [9], a dificuldade, a complexidade e a importância das questões.

Para tal fim, foram definidos cinco conjuntos difusos para cada fator de avaliação:

- Baixo (1)

- Baixo-Médio (2)

- Médio (3)

- Médio-Alto (4)

- Alto (5)

Nas seções seguintes apresentamos as tabelas construídas com as regras difusas definidas pelo especialista no domínio.

\section{Regras difusas para determinar a dificul- dade}

Neste caso foram construídas, pelo professor, as regras difusas para determinar a dificuldade dos alunos na resolução das atividades de acordo com o tempo gasto. A tabela 7 representa as regras difusas, que são a representação do conhecimento do professor especialista na disciplina e que servirão para determinar os pesos das questões de acordo com a dificuldade que os alunos tiveram em responder as atividades.

\begin{tabular}{|c|c|c|c|c|c|}
\hline \multirow{2}{*}{ Tempo } & \multicolumn{5}{|c|}{ Acerto } \\
\hline $\mathbf{1}$ & $\mathbf{1}$ & $\mathbf{2}$ & $\mathbf{3}$ & $\mathbf{4}$ & $\mathbf{5}$ \\
\hline $\mathbf{2}$ & 4 & 4 & 2 & 1 & 1 \\
\hline $\mathbf{3}$ & 4 & 4 & 2 & 1 & 1 \\
\hline $\mathbf{4}$ & 5 & 4 & 3 & 2 & 2 \\
\hline $\mathbf{5}$ & 5 & 5 & 3 & 2 & 3 \\
\hline
\end{tabular}

Tabela 7: Regras difusas para determinar a dificuldade.

\section{Regras difusas para determinar o esforço}

O esforço será determinado pela dificuldade que os alunos tiveram na resolução das atividades e a complexidade das mesmas. A tabela 8 representa as regras difusas para determinar os pesos das atividades considerando o esforço. Estas regras são construídas pelo professor especialista na disciplina.

\begin{tabular}{|c|c|c|c|c|c|}
\hline \multirow{2}{*}{ Complexidade } & \multicolumn{7}{c|}{ Dificuldade } \\
\hline $\mathbf{1}$ & $\mathbf{1}$ & $\mathbf{2}$ & $\mathbf{3}$ & $\mathbf{4}$ & $\mathbf{5}$ \\
\hline $\mathbf{2}$ & 2 & 2 & 3 & 3 & 3 \\
\hline $\mathbf{3}$ & 2 & 2 & 3 & 3 & 3 \\
\hline $\mathbf{4}$ & 3 & 3 & 3 & 4 & 4 \\
\hline $\mathbf{5}$ & 3 & 3 & 3 & 4 & 5 \\
\hline
\end{tabular}

Tabela 8: Regras difusas para determinar o esforço.

\section{Regras difusas para determinar o ajuste}

Finalmente, o ajuste de pesos atribuídos para cada atividade do módulo vai ser determinado pelo esforço e a importância das atividades no aprendizado do aluno.

Através de um processo retroativo o vetor de ajuste será utilizado para obter o vetor de pesos ajustados para cada atividade, determinando o desempenho dos alunos.

A tabela 9 representa as regras difusas para determinar o vetor de ajuste dos pesos das notas.

\begin{tabular}{|c|c|c|c|c|c|}
\hline \multirow{2}{*}{ Importância } & \multicolumn{7}{|c|}{ Esforço } \\
\hline $\mathbf{1}$ & $\mathbf{1}$ & $\mathbf{2}$ & $\mathbf{3}$ & $\mathbf{4}$ & $\mathbf{5}$ \\
\hline $\mathbf{2}$ & 2 & 2 & 3 & 3 & 3 \\
\hline $\mathbf{3}$ & 3 & 3 & 3 & 4 & 4 \\
\hline $\mathbf{4}$ & 3 & 3 & 3 & 4 & 5 \\
\hline $\mathbf{5}$ & 4 & 5 & 4 & 5 & 5 \\
\hline
\end{tabular}

Tabela 9: Regras difusas para determinar o ajuste dos pesos da nota.

\subsection{Avaliador Multidimensional Difuso}

Para desenvolver nossa proposta utilizamos o modelo de Saleh e Kim [7] implementado através do Fuzzy Logic Toolbox versão V2.2.11 (R2010a) da MathWorks (MATHWORKS).

\section{Controlador lógico difuso}

A inferência difusa é definida como um processo de formulação do mapeamento de uma dada entrada a uma saída usando a lógica difusa. O processo de inferência difusa envolve a utilização de funções de pertinência, operações lógicas (AND e OR) e estruturas de decisão (IF-THEN). O toolbox do Matlab oferece dois tipos de sistemas de inferência difusos: Mamdani e Sugeno. Estes sistemas de inferência se diferenciam na forma como são determinadas as saídas.

Em nossa proposta temos três controladores difusos. $\mathrm{O}$ primeiro calcula a dificuldade mediante os acertos e o tempo utilizado pelos alunos na resolução das atividades. O segundo calcula o esforço que é determinado pela dificuldade e a complexidades das questões. Por último, o 
ajuste final dos pesos das atividades é determinado pelo esforço do aluno e a importância das atividades do módulo.

Finalmente, as novas pontuações são determinadas pelo processo retroativo. Em outras palavras, a pontuação final de cada aluno é obtida mediante os acertos iniciais e os novos pesos obtidos por meio do processo difuso.

Cada controlador difuso é composto de cinco partes:

- Fuzzificação das variáveis de entrada (método triangular).

- Aplicação do operador difuso (AND ou OR) no antecedente (Inferência Mamadani’s).

- Implicação do antecedente para o consequente (Inferência Mamdani's).

- Agregação dos consequentes por meio das regras (Inferência Mamdani's).

- Defuzzificação (Centro de Gravidade).

Cada controlador difuso recebe duas entradas fuzzificadas utilizando o método triangular, posteriormente é inferido o resultado utilizando as regras difusas definidas pelo especialista no domínio, para finalmente defuzzificar os resultados aplicando o centro de gravidade (COG). A criação dos demais blocos de inferência difusa (esforço e desempenho) segue o mesmo procedimento.

\section{Avaliação}

Alimentando as entradas do nosso sistema difuso e tendo o motor de inferência configurado conforme as regras difusas obtidas, são inferidos os resultados da avaliação multidimensional. O sistema difuso também apresenta os resultados de uma avaliação tradicional (acerto/erro), sendo possível obter com maior exatidão o desempenho de cada aluno levando em consideração a dificuldade, complexidade e importância das questões.

As tabelas 10 e 11 apresentam os resultados finais do processo de avaliação difusa de cada uma das atividades realizadas, e também de forma tradicional.

No módulo educacional "Números Naturais" da quinta série, observa-se que o ALUNO7 e ALUNO8 não realizaram as atividades ATIV6 e ATIV7, tendo como valor de acerto igual a zero (vide tabela 2) e o tempo gasto igual a zero (vide tabela 3 ). Na avaliação tradicional os ALUNO7 e ALUNO8 (vide tabela 11) conseguem obter uma pontuação 30 de 100 pontos. Na avaliação multidimensional (vide tabela 10) os ALUNO7 e ALUNO8 obtiveram uma pontuação de 26,8644 pontos diminuindo em 3,1356 pontos em comparação com a avaliação tradicional. No entanto, o ALUNO5 que realizou a atividade ATIV6 e ATIV7 obteve 22,5 pontos na avaliação tradicional e somente diminuiu a pontuação em 0,4780 na avaliação multidimensional obtendo 22,0220 pontos. Finalmente os ALUNO2 e ALUNO9 obtiveram as maiores pontuações já que foram os únicos que acertaram as atividades ATIV5 e ATIV4 respectivamente, sendo estas as atividades de maior peso.

\begin{tabular}{|l|c|c|c|c|c|c|c|c|c|}
\hline \multicolumn{1}{|c|}{ AVALIAÇ̃̃O MULTIDIMENSIONAL } \\
\hline ALUNO1 & ALUNO2 & ALUNO3 & ALUNO4 & ALUNO5 & ALUNO6 & ALUNO7 & ALUNO8 & ALUNO9 & ALUNO10 \\
\hline 42,5363 & 68,6562 & 50,9259 & 35,2540 & 22,0220 & 24,8061 & 26,8644 & 26,8644 & 63,4322 & 42,5363 \\
\hline
\end{tabular}

Tabela 10: Avaliação multidimensional "Números Naturais da quinta série"

\begin{tabular}{|l|c|c|c|c|c|c|c|c|c|}
\hline \multicolumn{7}{|c|}{ AVALIAÇÃO TRADICIONAL } \\
\hline ALUNO1 & ALUNO2 & ALUNO3 & ALUNO4 & ALUNO5 & ALUNO6 & ALUNO7 & ALUNO8 & ALUNO9 & ALUNO10 \\
\hline 45,0000 & 70,0000 & 52,5000 & 37,5000 & 22,5000 & 27,5000 & 30,0000 & 30,0000 & 65,0000 & 45,0000 \\
\hline
\end{tabular}

Tabela 11: Avaliação tradicional "Números Naturais da quinta série"

\section{Conclusão}

Este trabalho pesquisou, implementou e aplicou o modelo de avaliação multidimensional baseado em lógica difusa de Saleh e Kim que contempla os três fatores de avaliação mencionados por Weon e Kim: importância, dificuldade e complexidade das questões. Pesquisamos, implementamos e aplicamos o modelo em um ambiente de ensino mediado por computador denominado por
Conexão do Saber utilizado na escola Aletheia, avaliando o desempenho dos alunos.

O processo de obtenção de dados reais, com professores e alunos, favoreceu os resultados da avaliação multidimensional, possibilitando a introdução de dados específicos, como taxa de acertos, tempo gasto pelos alunos em cada atividade, regras difusas, complexidade e importâncias das atividades atribuídas pelo professor. 
$\mathrm{O}$ uso da lógica difusa permitiu uma maior flexibilidade no momento de determinar o desempenho dos alunos, sendo possível mesurar o desempenho dos alunos utilizando os critérios definidos pelos professores e assim avaliar de uma maneira fácil e interpretativa. $\mathrm{O}$ resultado leva em conta diversas dimensões do aluno não se limitando as avaliações usuais.

O modelo de avaliação multidimensional baseado em lógica difusa para ambientes mediados por computador utilizado com sistema Conexão do Saber proporcionou uma vantagem sobre os sistemas tradicionais de ensino, já que os critérios de avaliação foram imersos no sistema e estes poderão ser alterados de acordo com a conveniência do avaliador.

A aplicação num ambiente de ensino mediado por computador pode permitir que as avaliações sejam feitas quase que instantaneamente após o término das atividades pelos alunos. É possível criar um histórico de avaliação para cada aluno que poderá ser utilizado pelo professor para analisar a evolução do aprendizado. Além do método levar em conta diversas dimensões, não apenas o acerto ou erro do exercício, a avaliação multidimensional aproxima o professor das atividades, pois a partir dele são criadas matrizes, como por exemplo, a de Importância e Complexidade. Estas matrizes não são estáticas, podendo ser atualizadas pelo professor à medida que este observa o comportamento dos alunos na resolução dos problemas. As matrizes de Acerto e Tempo podem ser geradas automaticamente pelo sistema, minimizando a intervenção do professor.

O modelo permite ainda a análise dos resultados obtidos na classificação tradicional utilizando a matriz de Ajuste que contém os novos pesos das atividades, permitindo ao professor reclassificar os resultados obtidos, ou seja, criamos uma multidimensionalidade sobre a avaliação tradicional.

\section{Referências}

[1] S. -M. Bai, S.-M. Chen, Automatically constructing grade membership functions of fuzzy rules for students' evaluation. Expert Systems with Applications: An International Journal, v. 35, n. 3, p. 1408-1414, 2008.

[2] S. -M. Bai, S.-M. Chen. Evaluating students' learning achievement using fuzzy membership functions and fuzzy rules. Expert Systems with Applications: An International Journal, v. 34, n. 1, p. 399-410, Janeiro 2008.

[3] A. C. G. Inocêncio. Processo otimizado para a produção de módulos Educacionais - Um estudo de caso do projeto Conexão do Saber. Tese de doutorado, Universidade Estadual de Campinas, Campinas, Março 2011.

[4] S. S. Lancaster, M. J. Wierman, Empirical study of defuzzification, Fuzzy Information Processing Society, 2003. NAFIPS 2003. 22nd International Conference of the North American, vol., no., pp.121,126, 24-26 July 2003.

[5] E. H. Mamdani, Application of Fuzzy Logic to Approximate Reasoning Using Linguistic Synthesis. IEEE Transactions on Computers, v. C26, n. 12, 1977.

[6] E. C. D. Oliveira, Ensino mediado pelo computador: novos papéis, novos desafios para professores de línguas estrangeiras. Revista Solta a Voz, v. 16, n. 1, p. 31-48, 2005.

[7] I. Saleh, S.-I. Kim, A fuzzy system for evaluating students' learning achievement. Expert Systems with Applications: An International Journal, Tarrytown, v. 36, n. 3, p. 6236-6243, Abril 2009.

[8] M. N. Sampaio, L. S. Leite, Alfabetização Tecnologica do Professor, Vozes, Petrópolis, RJ, páginas $111,2000$.

[9] S. Weon, J. Kim, Learning achievement evaluation strategy using fuzzy membership function. FIE '01 Proceedings of the Frontiers in Education Conference, 2001. on 31st, Washington, v. 1, p. 19-24, 2001

[10] L. A. Zadeh, Fuzzy sets. Information and Control, v. 8, n. 3, p. 338-353, 1965. 